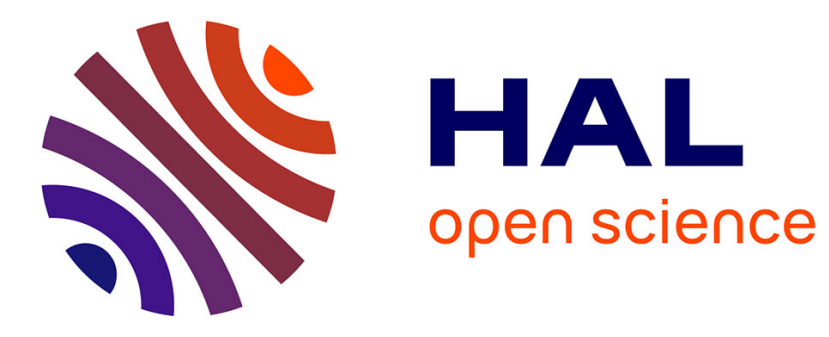

\title{
Exudate Segmentation on Retinal Atlas Space
}

Sharib Ali, Kedir Adal, Désiré Sidibé, T.P. Karnowski, M.D. Edward Chaum, Fabrice Meriaudeau

\section{To cite this version:}

Sharib Ali, Kedir Adal, Désiré Sidibé, T.P. Karnowski, M.D. Edward Chaum, et al.. Exudate Segmentation on Retinal Atlas Space. International Symposium on Image and Signal Processing and Analysis, Sep 2013, Trieste, Italy. pp.5. hal-00854653v2

\section{HAL Id: hal-00854653 \\ https://hal.science/hal-00854653v2}

Submitted on 9 Sep 2013

HAL is a multi-disciplinary open access archive for the deposit and dissemination of scientific research documents, whether they are published or not. The documents may come from teaching and research institutions in France or abroad, or from public or private research centers.
L'archive ouverte pluridisciplinaire HAL, est destinée au dépôt et à la diffusion de documents scientifiques de niveau recherche, publiés ou non, émanant des établissements d'enseignement et de recherche français ou étrangers, des laboratoires publics ou privés. 


\title{
Exudate Segmentation on Retinal Atlas Space
}

\author{
Sharib Ali*, Kedir M. Adal ${ }^{*}$, Désiré Sidibé *, Thomas P. Karnowski ${ }^{\dagger}$, Edward Chaum,M.D. ${ }^{\ddagger}$ \\ and Fabrice Mériaudeau* \\ *Laboratoire Le2i UMR CNRS 6306, Université de Bourgogne, Le Creusot, 71200, France \\ Email: ali.sharib2002@gmail.com \\ †Oak Ridge National Laboratory, Oak Ridge TN, USA \\ ${ }_{\ddagger}^{\ddagger}$ Hamilton Eye Institute, U. Tennessee Health Sciences Center, Memphis TN, USA
}

\begin{abstract}
Diabetic macular edema is characterized by hard exudates. Presence of such exudates cause vision loss in the affected areas. We present a novel approach of segmenting exudates for screening and follow-ups by building an ethnicity based statistical atlas. The chromatic distribution in such an atlas gives a good measure of probability of the pixels belonging to the healthy retinal pigments or to the abnormalities (like lesions, imaging artifacts etc.) in the retinal fundus image. Post-processing schemes are introduced in this paper for the enhancement of the edges of such exudates for final segmentation and to separate lesion from false positives. A sensitivity(recall) of $82.5 \%$ at $35 \%$ of positive predictive value on FROC-curve is achieved. Results are obtained on a publicly available HEI-MED data-set and have been compared to two reference methods on the same dataset showing the competitiveness of the proposed algorithm.
\end{abstract}

\section{INTRODUCTION}

\section{A. Clinical motivation}

Diabetic macular edema (DME) is one of the most severe complication seen in both pre-proliferative and proliferative diabetic retinopathy. According to the first global estimate of World Diabetes Population 2010, more than 21 million people worldwide are affected with it. In DME, vascular leakage produces yellow or white waxy patches often referred as "hard exudates". These are the hallmarks for the diagnosis of macular edema in fundus images. Macula is the most central area of the retina for vision. The adverse effect of presence of these exudates results from distorted vision to complete loss of vision. A regular follow-up and diagnosis can help in treatment of them by laser surgery and can reduce the risk of blindness by $90 \%$.

\section{B. Previous work}

Bright lesion segmentation (like exudates) can be divided into adaptive grey level based thresholding [1], region growing method [2], morphology based technique [3], and classification methods [4]-[6]. Sopharak et al. [3] proposed a method to automatically extract the exudates from the images of diabetic patients with non-dilated pupil. In their approach they used fuzzy $\mathrm{C}$-mean clustering followed by morphological closing reconstruction. Standard deviation, hue, intensity and number of edge pixels are selected as input features based on exudate characteristics. The algorithm was evaluated on 10 images against their ground truth manually obtained by ophthalmologists. They present an overall sensitivity of $87.2 \%$ at specificity of $99.2 \%$. Morphological closing reconstruction technique can give distance information between the detected exudates and the optic disc but it fails to detect tiny exudates. The algorithm also cannot separate the exudates and the imaging artifacts. Sanchez et al. [7] used the green channel of the fundus image due to its higher contrast in RGB color space. The authors found the pixels belonging to the background using the method described in [8] and applied bilinear interpolation to generate the complete background. The image is then modeled as a mixture of three Gaussian models representing background, foreground and outliers respectively. The foreground constitute vessels, optic disk and lesion. An EM (Expectation-Maximization) algorithm was used to estimate the exudate candidate and thresholded dynamically. As the exudate detection procedure, the authors employed Kirsch's edge operator [9] to separate the exudate from artifacts and other bright lesions. Niemeijer et al. [4] applied a machine learning approach for the automatic detection of exudates and cotton-wool spots in color fundus images. They presented a method for distinguishing among drusen, exudate and cotton wool spots. 300 retinal images of patients with diabetes were chosen from tele-diagnosis database. The gold standard was built on this dataset by two retinal specialists. They achieved $95 \%$ sensitivity at $88 \%$ specificity for the bright lesions. The sensitivity/specificity pairs were $77 \% / 88 \%, 95 \% / 86 \%$ and $70 \% / 93 \%$ for drusen, exudates and cotton wool spots detection respectively. Giancardo et al. [6] used the preprocessing step by background estimation with a large median filter on the I-channel of the normalized image in HSI color space. The normalized image was further enhanced with morphological reconstruction. This gave a clear distinction between the dark and bright structures. The author manually removed the optic nerve. Exudate candidates were selected based on some score using connected component analysis. The score was assigned based on Kirsch edges and stationary wavelets analysis. The method was evaluated using varying threshold from 0 to 1 in steps of $0.05(0: 0.05: 1)$. All of these classification methods require an accurate manual annotations for the training dataset. In addition they are also highly dependent on the type of features selected for classification.

\section{Aim of study}

The retinal image acquisition using low cost fundus camera is the most widely used technique. However, the segmentation of such bright lesions and their subsequent follow-ups are not easy because of 1) presence of anatomical structures with highly correlated pixels with that of lesion, 2) illumination variability and 3) movement of the eye during multiple visits of the patient. We propose an automatic statistical atlas based exudate segmentation algorithm. The test image is warped on to this atlas co-ordinate. A distance map with this atlas 


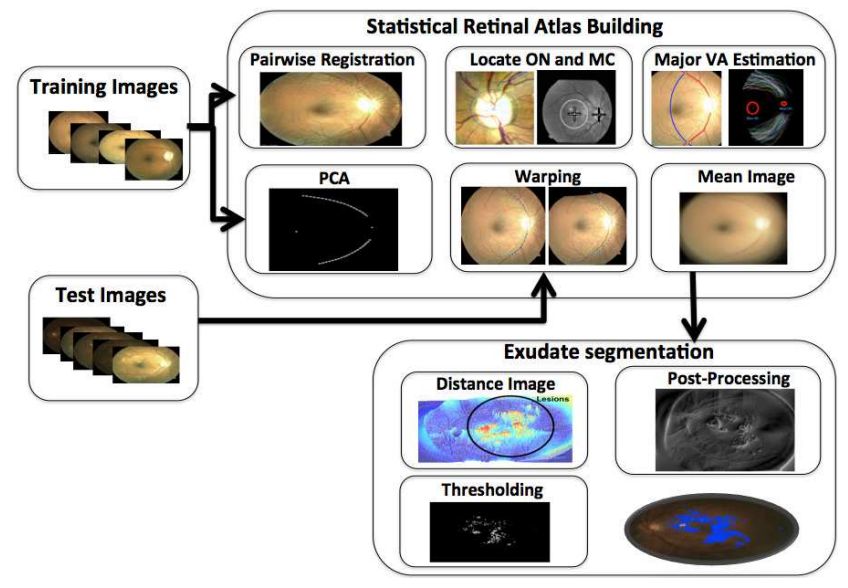

Fig. 1: Block diagram of overall process.

image not only suppresses the optic disc and other vasculatures in the retinal image but also registers the image to a common co-ordinate system. The lesions on this map show high deviation from the healthy retinal pixels. Further, postprocessing schemes are applied for the edge enhancement of these lesions. Statistical retinal atlas has been shown to be effective method for image grading [10] but has not been exploited for quantification of any kind of lesion segmentation.

\section{MATERIALS AND METHOdOLOGY}

The retinal atlas is created from 400 good quality paired fundus images of healthy African American eye from a dataset containing 5,218 retina fundus images collected from February 2009 to August 2011 from clinics in the mid-South region of the USA as part the Telemedical Retina Image Analysis and Diagnosis (TRIAD) project [11]. The images are from both healthy and abnormal retinas with color variations covering the pigmentation spectrum found in the patient population, which is approximately $70 \%$ African American and 30\% Caucasian. Exudate segmentation is validated using publicly available HEI-MED dataset [6] for diabetic macular edema. It includes a mixture of images with no macular edema or with varying degree of macular edema. The dataset consists of 169 fundus images with mixed ethnicities. We evaluate our methods with the 104 images corresponding to African American patients. The idea of building an ethnic group based statistical retinal atlas is because the ethnic background plays a significant role in retinal luminance in the fundus images [12].

The process includes the building of the statistical atlas using the images of the same ethnic group in the dataset mentioned above. Any test image with the same ethnicity patient is warped to the atlas space. A distance map is created and further post-processing schemes are used. We have used Kirsch's operator and Riesz transform. The idea behind using two edge detectors is to further enhance the edges found by the Riesz transform. The method is detailed below and is shown in Fig. 1.

\section{A. Statistical retinal atlas building}

A retinal atlas provides a reference representation for important retinal structures: major vessel arches (superior and inferior), optic disc, fovea and eye pigmentation. First, a reference co-ordinate system is identified by rigid alignment of the detected optic nerve center, macula center and by finding mean shape of the tracked major vessel arches in the training dataset. The overall process is discussed below:

1) Paired retinal images registration: The paired images are first registered using a feature based registration method. Feature vectors are extracted from the intensity image using SURF algorithm resulting in a 64-dimensional feature vector for each interest point [13]. An interest point in the test image is compared to an interest point in the target image by calculating the Euclidean distance between their respective descriptor vectors. A matching pair is detected, if its distance is closer than 0.7 times the distance of the second nearest neighbour. This is the nearest neighbour ratio matching strategy used to eliminate ambiguous pair of features [14]. We then apply RANSAC algorithm to further remove outliers and estimate the best transformation parameters from the known matches.

2) Vessel arch estimation: Considering the automatically detected optic disk location using Hough transform [15] as center of an arbitrary co-ordinate system shown in Fig. 2(a), we define some empirical assumptions for finding the major arches in the registered image pairs of each eye. These assumptions are used in locating the seed points for the vessel arches, which are then found automatically. For the right eye, starting from the detected optic disc center, the search is made in the interval $\left[90^{\circ}, 135^{\circ}\right]$ for the upper arch and in $\left[225^{\circ}, 270^{\circ}\right]$ for the lower arch. Similarly, for the left eye, the search is made in the closed interval $\left[45^{\circ}, 90^{\circ}\right]$ for the upper arch while for the lower arch the interval is $\left[270^{\circ}, 315^{\circ}\right]$.

A rectangular mask of size $20 \times 20$ is taken around each pixel, considering the maximum diameter of the major arches to be not more than 20 pixels, within the search interval mentioned above to find the highly correlated pixels using a low pass filter and a differentiator correlation kernel on vessel enhanced image. Vessel enhancement was done by analysing the eigen system of the Hessian matrix according to [16]. The correlation filter [17] adapted on this filtered image is locally oriented along $\mathrm{x}$-axis and is defined by,

$$
\begin{aligned}
h(x, y)= & \frac{1}{8 K}\{-2 \delta(y-2)-2 \delta(y-1)+2 \delta(y+1) \\
& +2 \delta(y+2)\} \otimes\left\{\sum_{k=0}^{K-1} \delta(x-k)\right\},
\end{aligned}
$$

where $\otimes$ represents the convolution operator along y-direction, $\delta$ represents the impulse response of the filter and $K$ is the kernel size.

The pixels within the constrained search window defined are convolved with the kernel in equation 1 . The peak response of this correlation kernel is obtained at the major vessel arches. The pixel location is taken as a seed point for tracking the major vessel. Vessel boundaries can be obtained by rotating correlation kernels given by equation 1. Multiple templates are correlated with the seed point and the search is moved along the point with the highest response [17]. Each template is divided into right and left template. Left template finds the edge location in $90^{\circ}$ counter-clockwise direction and right is tuned to the right boundary. The angle of rotation of the kernel is discretized into 16 values at spacing of $22.5^{\circ}$ all with the 


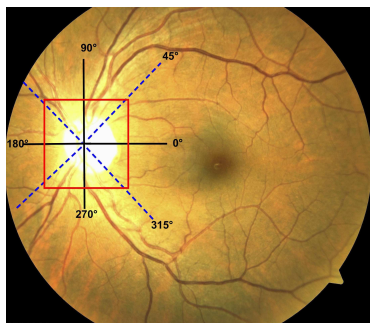

(a)

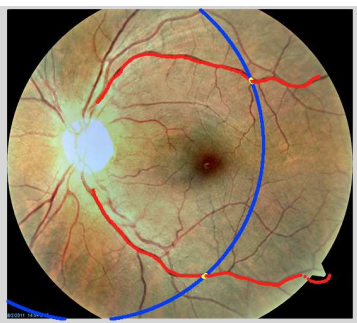

(b)
Fig. 2: Vessel Tracking in Left eye. a) Search location of major arches; b) Automatic major arches tracing. Red points are the traced path and blue curve is represents the estimation of the end points of these major arches.

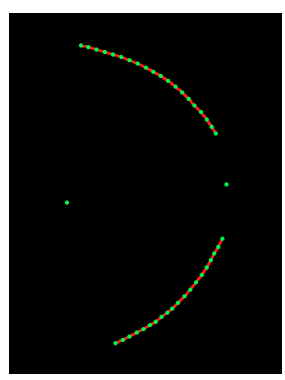

(a)

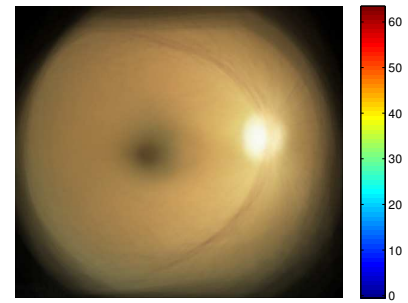

(b)
Fig. 3: Statistical atlas. a) Atlas co-ordinate frame; b) Mean atlas image.

same sum of square weights of 60 for each template. The tracked major arches are shown in Fig. 2(b). The blue arc gives the end points cutting the tracked vessels at yellow points. Typically, the radius of this arc is taken equals to $1.45 \times$ the distance from macula center to optic disk for both right eye and left eye images. The images in the dataset used are macula centered. The optic disc localization was done using circular Hough transform [15].

3) Warping and atlas: The vessel arches are estimated for $N$ images. We then automatically find $M(M=20)$ equidistant points on each of the two major arches as explained above. PCA (principal component analysis) is applied on these points to find the two mean major vessel arches forming an atlas co-ordinates frame along with the mean macula center and mean optic disc center as shown in Fig. 3(a). The images used were macula centered so we empirically assume it to be at the center of the image.

Each training image is warped onto this atlas co-ordinate frame using thin-plate splines. After all the images are warped into this atlas co-ordinate system, we take the mean to get the average color/pigmentation in the population. The mean image is normalized to obtain an atlas map for the ethnic group (here African American) population as shown in Fig. 3(b).

\section{B. Exudate Segmentation}

The test image belonging to the same ethnic group as that of the atlas in use is first warped onto the atlas co-ordinate system. Then a distance map is obtained with that of the mean

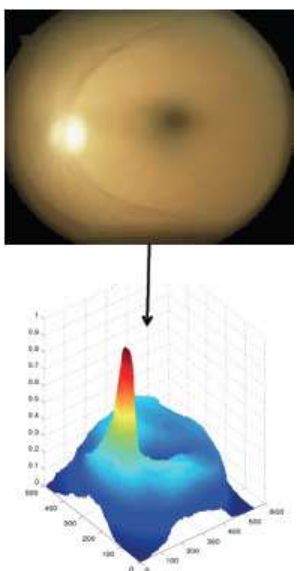

(a)

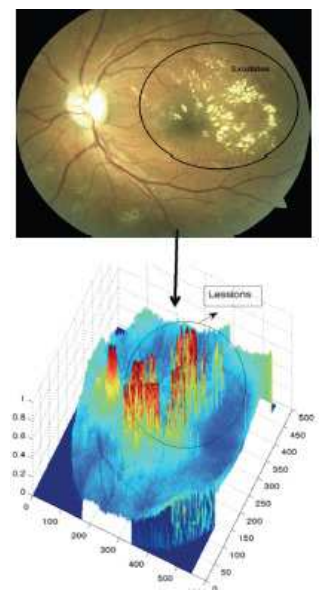

(b)

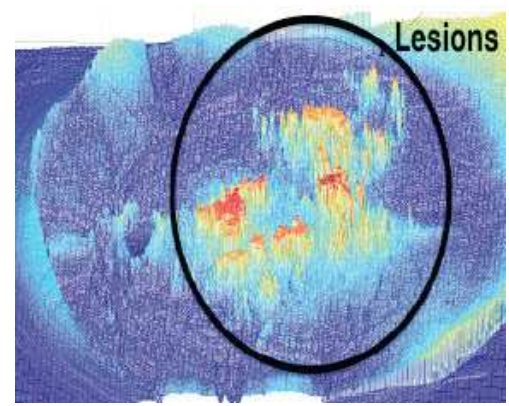

(c)

Fig. 4: Segmentation of exudates on atlas space. a) Atlas mean image and mesh plot; b) Unhealthy eye (exudates) and mesh plot ; c) Distance image between a) and b).

atlas image. This suppresses the similar pixels located at the same positions as shown in Fig. 4. However, the pixels having high variance between the test image and the mean atlas image are those belonging to lesions or having high probability of being lesion like imaging artifacts or drusen or cotton-wool spots. To eliminate such false positive (FP) detections we used two post-processing schemes. Riesz transform was used to enhance the remaining structures that are not suppressed then Kirsch edge operator was used to enhance only those pixels that belonged to exudates. Our experiments showed that using the combination of these two post-processing schemes eliminated the FPs.

Riesz transform is a multidimensional extension of the Hilbert transform. The space-domain representation of $d-$ channel filterbank is given by:

$$
\widehat{\boldsymbol{R} f(x)}=\left(\begin{array}{c}
R_{1} f(x) \\
\cdot \\
\cdot \\
R_{d} f(x)
\end{array}\right) \leftrightarrow^{\mathrm{F}}-j \frac{w}{\|w\|} \hat{f}(x), \forall f \in L_{p}\left(\Re^{d}\right) .
$$

The component filters of the Riesz transform in Eq. (2) are $90^{\circ}$ rotated versions of each other. A general representation of filterbank response that can be steered in any direction is presented as, 


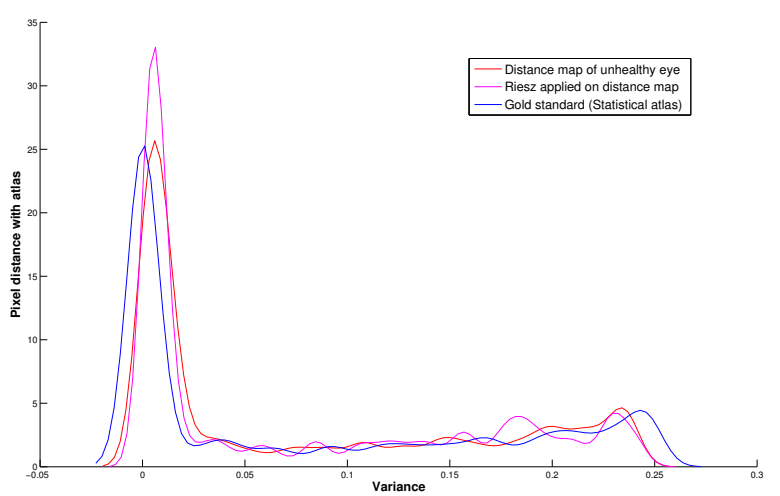

Fig. 5: Post processing scheme enhancing the lesion pixels in an unhealthy test image [21] .

$$
H_{\boldsymbol{u}} f(x)=\sum_{n=1}^{d} u_{n} R_{n} f(\boldsymbol{x})=<\boldsymbol{u}, \boldsymbol{R} f(\boldsymbol{x})>
$$

One of the major advantage of using Riesz tansform is that the coefficients are essentially zero in smooth area of the image [18] and has been exploited for texture enhancement in past [19].

Kirsch operator is a non-linear edge detector kernel that evaluates the edges in 8 different directions on an image. The operator is calculated for 8 directions with $45^{0}$ difference [20].

$$
h_{n, m}=\max _{z=1, \ldots, 8} \sum_{i=-1}^{1} \sum_{j=-1}^{1} p_{i j}^{(z)} \cdot I_{n+i, m+j},
$$

where $p_{i j}^{(z)}$ is the directional kernel.

The result of the post processing scheme using Riesz transform [21] is shown in Fig. 5. We can clearly notice that the post-processing scheme increases the cluttering of the lesion pixels thus making the thresholding easier.

A soft thresholding is done after the treatment with the post-processing scheme presented. The thresholding values were empirically set as $(0: 0.1: 1)$. The results of the exudate segmentation using the statistical atlas and the post-processing schemes discussed above are shown in Fig. 6.

\section{RESULTS}

The accuracy of the performance of the diagnosis by an algorithm is characterized by plotting ROC curve and FROC curve. The area under the curve gives the accuracy of the algorithm in use. However, when dealing with highly irregular datasets, Precision-Recall (PR) curves give a more informative picture of an algorithm's performance and there exists very strong connection between ROC space and PR space [23].

For our evaluation purpose with the ground truth provided in the HEI-MED dataset, a true positive is considered when at least a part of the lesion overlaps with the ground truth. The detection is a false positive when the exudate is found

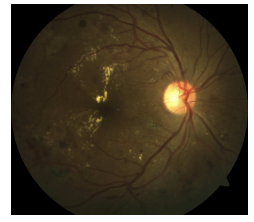

(a)

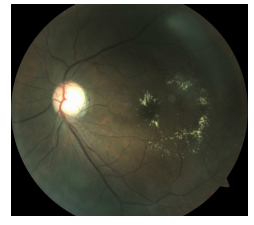

(d)

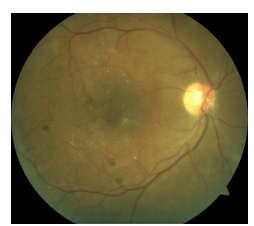

(g)

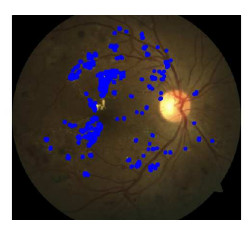

(b)

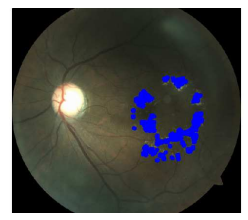

(e)

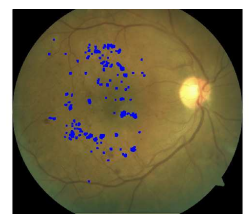

(h)

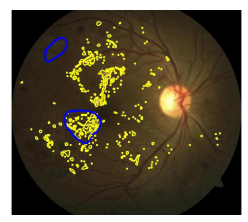

(c)

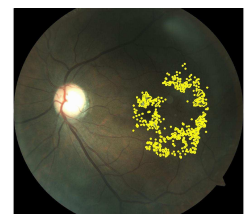

(f)

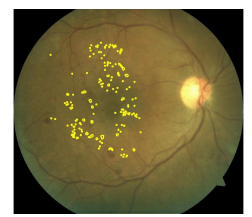

(i)
Fig. 6: Results of exudate segmentation: On the left column $(a, d, g)$ are original images; in middle column $(b, e, h)$ are images with labelled exudates from the output of the algorithm (thres=0.6) and on the right column $(c, f, i)$ are the ground truths annotated by an ophtalmologist [22]. On the topmost right column (c), the blue circles represent bright lesions which might not be possibly hard exudates.

outside the region of the manually annotated ground truth and a false negative if no lesion is found in the image while a lesion exists. Convex hulls in PR curves is achievable and is similar to the convex hulls in the ROC curve. We have used PR-curve but with the recall or sensitivity on the y-axis and precison or positive predictive value on the $\mathrm{x}$-axis. We call this curve "Free-Response ROC Curve" in this article since there is no widely accepted summary and system to evaluate the FROC model. The method explained in [23] has been used to accurately calculate the area under the curve (AUC).

The algorithm has been evaluated by comparing it with the other two best methods in the literature Sanchez et. al. (2009) [7] and Giancardo et. al. (2011) [6] for DME segmentation. We found our method to be promising as shown in Fig. 7. The major limitation during this evaluation was the lack of ground truth on the atlas space. So we had to unwarp the distance image back to the original space for evaluation. During the interpolation process there is always few pixels of error in the unwarping. Despite this disadvantage we obtained comparable results as shown in table I; without the need of removing the optic nerve manually as in [6] or removing the background as in [7]. The proposed atlas based exudate segmentation method achieves an accuracy of $82.60 \%$. This result should not be confused with the detection rate accuracy used for diagnosis of the patient which means finding 1 significant lesion. We are not presenting the ROC curve here because we are concerned with the overall exudate segmentation. However, we are confident that this approach can help in better candidate selection for the automatic diagnosis of the patients with diabetic macular edema. 


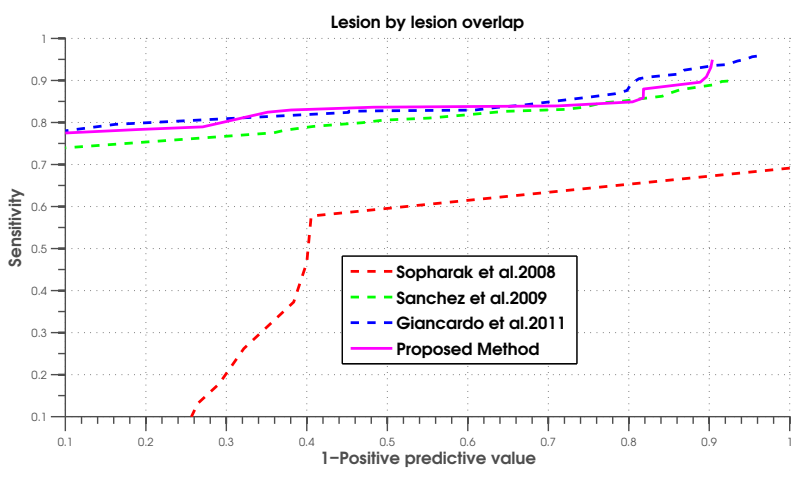

Fig. 7: FROC curve for evaluation of the method.

\begin{tabular}{|l|c|}
\hline Method & AUC \\
\hline \hline Sopharak et al. (2008) & 0.580 \\
\hline Sanchez et al. (2009) & 0.795 \\
\hline Giancardo et al. (2011) & 0.834 \\
\hline Proposed Method & $\mathbf{0 . 8 2 6}$ \\
\hline
\end{tabular}

TABLE I: AUC comparison (methods in literature)

\section{CONCLUSION}

The significance and the utility of the statistical atlas for exudate segmentation has been presented in this paper. It has been shown that bright lesions segmentation onto the atlas space can be done without doing pre-processing steps like image normalization, optic disc and vessel removal etc. Experiments showed that lesions are more apparent relative to the background vasculatures in the distance image on the atlas space. The results presented in this paper proved the reliability of this method. The proposed method can potentially be used for the follow-up visits as all the test images are taken to one coordinate frame (i.e. atlas co-ordinate system). This will help in the analysis of the area covered by the lesions during subsequent examinations.

\section{ACKNOWLEDEMENT}

This work has been conducted in collaboration between University of Burgundy, France and Oak Ridge National Laboratory, USA. We would like to thank the "Regional Burgundy Council" for co-sponsoring the work.

\section{REFERENCES}

[1] R. Phillips, J. Forrester, and P. Sharp, "Automated detection and quantification of retinal exudates," Graefe's Archive for Clinical and Experimental Ophthalmology, vol. 231, pp. 90-94, 1993.

[2] C. Sinthanayothin, J. F. Boyce, T. H. Williamson, H. L. Cook, E. Mensah, S. Lal, and D. Usher, "Automated detection of diabetic retinopathy on digital fundus images." Diabetic medicine : a journal of the British Diabetic Association, vol. 19, pp. 105-112, 2002.

[3] A. Sopharak, B. Uyyanonvara, and S. Barman, "Automatic exudate detection from non-dilated diabetic retinopathy retinal images using fuzzy c-means clustering," Sensors, vol. 9, no. 3, pp. 2148-2161, 2009.

[4] M. Niemeijer, B. van Ginneken, S. R. Russell, M. S. A. SuttorpSchulten, and M. D. Abramoff, "Automated detection and differentiation of drusen, exudates, and cotton-wool spots in digital color fundus photographs for diabetic retinopathy diagnosis," Invest. Ophthalmol. Vis. Sci., vol. 48, no. 5, pp. 2260-2267, May 2007.
[5] M. García, C. I. Sánchez, M. I. López, D. Abásolo, and R. Hornero, "Neural network based detection of hard exudates in retinal images," Comput. Methods Prog. Biomed., vol. 93, no. 1, pp. 9-19, Jan. 2009.

[6] L. Giancardo, F. Meriaudeau, T. P. Karnowski, Y. Li, K. W. Tobin, and E. Chaum, "Automatic retina exudates segmentation without a manually labelled training set," in ISBI, 2011, pp. 1396-1400.

[7] C. I. Sánchez, M. García, A. Mayo, M. I. López, and R. Hornero, "Retinal image analysis based on mixture models to detect hard exudates." Medical Image Analysis, vol. 13, no. 4, pp. 650-658, 2009.

[8] M. Foracchia, E. Grisan, and A. Ruggeri, "Luminosity and contrast normalization in retinal images." Medical Image Analysis, vol. 9, no. 3, pp. 179-190, 2005.

[9] R. A. Kirsch, "Computer determination of the constituent structure of biological images," Computers and Biomedical Research, pp. 315-328, 1970.

[10] S. Lee, M. D. Abramoff, and J. M. Reinhardt, "Retinal atlas statistics from color fundus images," in Proc. SPIE Conf. Medical Imaging, B. M. Dawant and D. R. Haynor, Eds., vol. 7623, 2010, p. 762310.

[11] Y. Li, T. Karnowski, K. Tobin, L. Giancardo, S. Morris, S. Sparrow, S. Garg, K. Fox, and E. Chaum, "A health insurance portability and accountability act-compliant ocular telehealth network for the remote diagnosis and management of diabetic retinopathy," Telemedicine and e-Health, 2011.

[12] R. R. A. Bourne, "Ethnicity and ocular imaging," Eye, vol. 25, pp. 297-300, 2011.

[13] H. Bay, A. Ess, T. Tuytelaars, and L. Van Gool, "Speeded-up robust features (surf)," Comput. Vis. Image Underst., vol. 110, no. 3, pp. 346359, 2008.

[14] D. G. Lowe, "Distinctive image features from scaleinvariant keypoints," Int. J. Comp. Vis, pp. 91-110, 2004.

[15] M. Blanco, M. G. Penedo, N. Barreira, M. Penas, and M. J. Carreira, "Localization and extraction of the optic disc using the fuzzy circular hough transform," in Proceedings of the 8th international conference on Artificial Intelligence and Soft Computing, ser. ICAISC'06. Berlin, Heidelberg: Springer-Verlag, 2006, pp. 712-721.

[16] A. Frangi, W. Niessen, K. Vincken, and M. Viergever, "Multiscale vessel enhancement filtering," 1998, pp. 130-137.

[17] A. Can, H. Shen, J. N. Turner, H. L. Tanenbaum, and B. Roysam, "Rapid automated tracing and feature extraction from retinal fundus images using direct exploratory algorithms," IEEE Transactions on Information Technology in Biomedicine, vol. 3, no. 2, pp. 125-138, 1999.

[18] M. Unser and D. Van De Ville, "Wavelet steerability and the higherorder Riesz transform," IEEE Transactions on Image Processing, vol. 19, no. 3, pp. 636-652, March 2010 .

[19] A. Depeursinge, A. Foncubierta-Rodriguez, D. Van de Ville, and H. Müller, "Lung texture classification using locally-oriented riesz components," in Proceedings of the 14th international conference on Medical image computing and computer-assisted intervention - Volume Part III, ser. MICCAI'11, 2011, pp. 231-238.

[20] R. A. Kirsch, "Computer determination of the constituent structure of biological images," Computers and Biomedical Research, pp. 315-328, 1970.

[21] S. Ali, K. M. Adal, D. Sidibé, E. Chaum, T. P. Karnowski, and F. Mériaudeau, "Steerable wavelet transform for atlas based retinal lesion segmentation," Proc. SPIE, Medical Imaging: Image Processing, vol. 8669, pp. 86693D-10, 2013.

[22] L. Giancardo, F. Meriaudeau, T. P. Karnowski, Y. Li, S. Garg, K. W. Tobin, and E. Chaum, "Exudate-based diabetic macular edema detection in fundus images using publicly available datasets." Medical Image Analysis, vol. 16, pp. 216-226, 2012.

[23] J. Davis and M. Goadrich, "The relationship between precision-recall and roc curves," in Proceedings of the 23rd international conference on Machine learning. ACM, 2006, pp. 233-240. 